\title{
DEPOSIÇÃO DE ESGOTO DOMÉSTICO PARA CONTROLE DE POLUIÇÃO E REVEGETAÇÃO INDUZIDA EM ÁREA DEGRADADA
}

\author{
Rodrigo Studart Corrêa ${ }^{1}$, Benício de Melo Filho ${ }^{2}$ \& Ricardo S. Bernardes ${ }^{3}$
}

\begin{abstract}
RESUMO
Este trabalho testa o comportamento ao longo de 2 anos de um Sistema de Infiltração Rápida-SIR, construído sobre uma área escavada em Latossolo Vermelho-escuro, na Bacia do Lago Paranoá, Distrito Federal-DF. Fósforo é atualmente o nutriente que mais contribui para o perigo de eutrofização desse reservatório. Por outro lado, os Latossolos do DF são deficientes desse nutriente, apresentam alta capacidade de fixação de fósforo e de infiltração de água. Os locais minerados no DF necessitam de grande aporte de matéria orgânica para sustentarem vegetação. O SIR permitiu o estabelecimento de diversas espécies das famílias Graminae, Melastomataceae e Compositae. A concentração de fósforo aumentou 84 vezes no solo entre $5-10 \mathrm{~cm}$ de profundidade. Mais de $10 \mathrm{t}$ de matéria orgânica e cerca de $41 \mathrm{~kg}$ de fósforo, antes despejados no Lago, foram mantidos no solo. O SIR alcançou uma eficiência de $88,3 \%$ na remoção de fósforo e mostrou-se promissor como alternativa à disposição de esgotos em corpos d'água e na indução do estabelecimento de vegetação sobre locais minerados.
\end{abstract}

Palavras-chave: esgotos, fósforo, áreas degradadas, revegetação, recuperação

\section{WASTEWATER DISPOSAL FOR POLLUTION CONTROL AND INDUCING REVEGETATION ON A BARREN LAND}

\begin{abstract}
This paper evaluates the behaviour of a Rapid Infiltration System (RIS) working on a $1.250 \mathrm{~m}^{2}$ mined plot of Dark Redish Latosol in the Paranoá Lake catchment area (Brasília City, Brazil) over 2 years. Phosphorus is the current nutrient that poses the highest eutrophication risk to that water reservoir. On the other hand, the local soils are very poor in it and they present high phosphorus fixing capacity and high water infiltration capacity too. Furthermore, the stripped sites can not be rehabilitated without high inputs of organic matter. The implementation of the RIS induced the establishment of several species belonging to the Graminae, Melastomataceae, and Compositae families. The phosphorus concentration increased 84 times in the $5-10 \mathrm{~cm}$ soil layer. Over 10 tonnes of organic matter and nearly $41 \mathrm{~kg}$ of phosphorus previously disposed into the Lake were kept in the soil. The RIS presented $88.3 \%$ of efficiency on phosphorus removal and was very successful for inducing site reclamation.
\end{abstract}

Key words: sewage, wastewater disposal, phosphorus, barren lands, reclamation

Recebido em 20/01/2000, Protocolo 007/00

${ }^{1}$ Bolsista de Doutorado do CNPq e Eng. Agrônomo da SEMARH/DF, The University of Melbourne-ILFR, Victoria 3010 , Austrália.E-mail:r.correa@pgrad.unimelb.edu.au

${ }^{2}$ Mestrando do Centro de Desenvolvimento Sustentável da Universidade de Brasília-UnB/CDS e Eng. Florestal do IBAMA, CP 10814, CEP 70300 - 980, Brasília, DF. E-mail: fbenicio@unb.br

${ }^{3}$ Professor do Depto. de Engenharia Civil da Universidade de Brasília-UnB/FT/ENC, Campus Universitário Darcy Ribeiro, CEP 70910 - 900, Brasília, DF. E-mail: ricardo@guarany.cpd.unb.br 


\section{INTRODUÇÃO}

O esgoto doméstico de aproximadamente 400 residentes do Centro Olímpico da Universidade de Brasília, fôra despejado por cerca de 35 anos no Lago Paranoá, após passar por uma fossa séptica. O Lago Paranoá é um reservatório artificial de 40 $\mathrm{km}^{2}$ de área e $14 \mathrm{~m}$ de profundidade média a bacia do Paranoá é a mais povoada do Distrito Federal, contando, atualmente, com aproximadamente 1 milhão de habitantes. Cerca de $85 \%$ do volume de esgotos do Distrito Federal são tratados e o restante geralmente é coletado em fossas sépticas, com parte despejada em cursos d'água e lagos.

O principal problema associado ao Lago é a poluição causada pela alta carga de matéria orgânica e nutrientes recebidos. Cerca de $100 \mathrm{~kg}$ de fósforo são diariamente despejados no Lago e sua capacidade de receber esse nutriente, antes que ocorram sérios riscos de eutrofização, é de apenas mais $12 \mathrm{~kg} \mathrm{dia}^{-1}$. Nitrogênio e fósforo são os principais nutrientes que determinam os riscos e níveis de eutrofização em águas superficiais (Loehr, 1979). Apesar de o nitrogênio existir em esgotos em concentrações mais altas (15-85 $\left.\mathrm{mg} \mathrm{L}^{-1}\right)$ que o fósforo, este representa maior risco a longo prazo. A fixação de fósforo por sedimentos minerais e matéria orgânica faz com que ele se acumule nos sistemas lacustres.

O principal objetivo do tratamento de esgotos é proteger, de maneira econômica e socialmente aceitável o meio ambiente e a saúde pública (Metcalf \& Eddy, 1991). A utilização benéfica de esgotos encontra-se entre uma das prioridades do manejo de resíduos em todo o mundo (Epstein, 1997). O interesse em sistemas naturais de tratamento de esgotos baseia-se na ética de utilização, reutilização e reciclagem de resíduos, sempre que possível (Reed et al., 1995).

Tratamentos que usam o solo como meio de depuração de esgotos combinam mecanismos físicos, químicos e biológicos. De acordo com Metcalf \& Eddy (1991), a qualidade dos efluentes que deixam os solos é similar, ou melhor, que aqueles provenientes de estações mecânicas de tratamento. Alguns solos são capazes de remover até $99 \%$ do fósforo, nitrogênio, coliformes totais, sólidos em suspensão e demanda bioquímica de oxigênio presentes no esgotos (Nucci et al., 1978; USEPA, 1981); o processo é melhor quando há uma vegetação estabelecida no local (Nucci et al., 1978) e, de acordo com Couracci Filho et al. (1996), os parâmetros dos efluentes originados de sistemas de infiltração em solos são compatíveis com a legislação brasileira.

A Agência Norte-americana de Proteção Ambiental (USEPA, 1984) cita que solos de textura argilosa podem ser utilizados em sistemas de infiltração de esgotos, caso apresentem características apropriadas. Os Latossolos vermelho-escuro estendem-se pela maior parte da Bacia do Paranoá. Apesar de muito argilosos (60-80\% de argila), sua estrutura lhes permite taxas de infiltração que variam entre $17-22 \mathrm{~cm} \mathrm{~h}^{-1}$ (Corrêa \& Leite, 1998; Resk, 1981).

Adsorção e precipitação são os principais mecanismos de remoção de fósforo do meio líquido quando esgotos filtram através de solos (Reed et al., 1995). Assume-se que componentes fosfatados serão retidos pelas partículas de solo e, por isso, curvas de adsorção de fósforo são geralmente determinadas para a construção e tempo de operação desses sistemas (Skilton et al., 1998). A contínua disposição de esgoto no solo pode causar excessiva concentração de fósforo, atenuada ou evitada quando há vegetação presente. De qualquer forma, há sistemas operando desde 1897, em Werribee (Austrália) e 1963, na Pennsyilvânia (USA) que ainda não apresentaram declínio na capacidade de remoção de fósforo dos influentes. A produção intensa de culturas vegetais nessas áreas é um dos fatores que mais contribuem para essa longevidade.

Existem dezenas de cascalheiras (Cambissolos) e áreas de empréstimo (Latossolos) na Bacia do Paranoá, originadas em 40 anos de obras civis. Inúmeras tentativas de revegetação desses locais falharam, principalmente pelo baixo teor de matérica orgânica nesses substratos (Correa \& Melo Filho, 1998). De acordo com Dedecek (1993), tais áreas necessitam de grande quantidade de matéria orgânica para suportarem uma cobertura vegetal.

A alta capacidade de fixação de fósforo, aliada aos valores extremamente baixos desse elemento disponível na solução do solo, é uma das grandes limitações à produção vegetal em Latossolos no Cerrado, por questões econômicas (Lopes, 1983). Os altos teores de sesquióxidos de ferro e alumínio dos Latossolos fazem deles grandes fixadores de fósforo, regra válida para outras partes da América Tropical. De acordo com Malavolta \& Kliemmann (1985), 93\% da área total da América Tropical são cobertos por solos deficientes em fósforo e $74 \%$ por solos que apresentam alta fixação desse elemento. Lopes (1983), estudando solos no Cerrado, concluiu que para cada 0,2 ppm de fósforo disponível na solução, há entre 0,4 e 2,4 mg P kg-1 fixados no solo.

Para garantir a proteção de manaciais subterrâneos, alguns regulamentos especificam que as taxas de aplicação de esgostos nos solos devem basear-se preferencialmente na quantidade de fósforo presente. Como esgotos contêm menos fósforo (3-15 $\left.\mathrm{mg} \mathrm{L}^{-1}\right)$ que nitrogênio $\left(15-85 \mathrm{mg} \mathrm{L}^{-1}\right)$ e a maioria das espécies vegetais necessita mais de nitrogênio que de fósforo, existe um fator de segurança inerente nessa abordagem (ASCE, 1987; Metcalf \& Eddy, 1991; Sedlak, 1991).

O presente trabalho objetiva testar o comportamento de um Sistema de Infiltração Rápida construído sobre o horizonte C de um Latossolo Vermelho-escuro quanto à resposta da área ao aporte de esgoto e à eficiência na remoção de fósforo do influente, após dois anos de operação ininterrupta. Este manejo nunca fôra testado no Distrito Federal e contrasta com o atual cenário predominante, que pode ser resumido em:

$$
\text { esgoto bruto } \rightarrow \text { fossa séptica } \rightarrow \text { cursos d'água }
$$

O cenário alternativo proposto e testado neste trabalho pode ser resumido em:

esgoto bruto $\rightarrow$ fossa séptica $\rightarrow$ sistemas de infiltração em
áreas degradadas

\section{MATERIAL E MÉTODOS}

Uma área de aproximadamente $2.000 \mathrm{~m}^{2}$ de Latossolo vermelho-escuro, desprovido dos horizontes superficiais A e $\mathrm{B}$, foi selecionada perto da descarga do efluente da fossa séptica do Centro Olímpico (CO) da Universidade de Brasília (UnB). 
Pelos critérios de Reed et al. (1995), que consideram profundidade do solo, taxa de infiltração, ausência de camada de impedimento, declividade e clima, as condições do local selecionado podem ser classificadas como ideais para operar um Sistema de Infiltração Rápida de esgotos.

Sulcos de $15 \mathrm{~cm}$ de profundidade e $20 \mathrm{~cm}$ de largura foram construídos em $1.250 \mathrm{~m}^{2}$ da superfície exposta, em forma de escama de peixe, para receberem até $60 \mathrm{~m}^{3}$ esgoto dia $^{-1}$. O efluente da fossa séptica, anteriormente despejado no Lago Paranoá, foi desviado para a parte superior do sulco principal, de onde passou a ser distribuído por gravidade. Para o horizonte C com $67 \%$ de argila utilizado neste estudo, considerou-se o valor de $1,7 \mathrm{mg}$ $\mathrm{kg}^{-1}$ de fósforo fixado, para cada $0,2 \mathrm{ppm}$ de fósforo disponível na solução do solo (Lopes, 1983).

Passados dois anos de contínua operação, 10 subamostras de solo de cada camada $0-5 \mathrm{~cm}, 5-10 \mathrm{~cm}$ e $10-20 \mathrm{~cm}$ foram coletadas com um trado agrícola na área irrigada e em porções da área que não receberam esgoto (controle). As subamostras foram misturadas considerando-se profundidade e tratamento, e deixadas a secar à sombra, por duas semanas. As 6 amostras compostas foram passadas em peneira de $2,0 \mathrm{~mm}$ e analisadas em triplicata.

A matéria orgânica dos substratos foi analisada oxidando-se $0,2 \mathrm{~g}$ de solo com um mistura de dicromato de potássio $\left(\mathrm{K}_{2} \mathrm{Cr}_{2} \mathrm{O}_{4}\right)$ e ácido sulfúrico $\left(\mathrm{H}_{2} \mathrm{SO}_{4}\right)$ e posterior titulação com sulfato ferroso aquoso $\left(\mathrm{FeSO}_{4} \cdot 7 \mathrm{H}_{2} \mathrm{O}\right)$. Cálcio $(\mathrm{Ca})$, magnésio $(\mathrm{Mg})$, potássio $(\mathrm{K})$, zinco $(\mathrm{Zn})$ e cobre $(\mathrm{Cu})$ foram analisados de acordo com EMBRAPA (1979); o fósforo foi extraído com uma mistura de ácido clorídrico $(\mathrm{HCl})$ e ácido sulfúrico $\left(\mathrm{H}_{2} \mathrm{SO}_{4}\right)$ (extrator Melich I). Os extratos foram analisados em espectofotômetro de absorção atômica a $660 \mathrm{~nm}$. Os resultados foram tratados por meio do teste de variância e as médias comparadas usando-se o teste de Tukey, a 5\% de significância.

\section{RESULTADOS E DISCUSSÃO}

A simples deposição do esgoto na área minerada proporcionou um ambiente favorável ao rápido estabelecimento de uma comunidade vegetal. As espécies presentes compunham-se principalmente de gramíneas pioneiras, Ricinus comunis, ervas, árvores e arbustos nativos das famílias Melastomataceae e Compositae. A disposição continuada do esgoto sobre o substrato exposto foi favorável ao desenvolvimento de sementes e propágulos que por meios próprios chegaram ali. A farta disponibilidade de água e a fertilização são as mais prováveis causas dessa revegetação natural, tendo em vista que outros elementos não foram mudados, acrescentados nem retirados do local. A concentração de nutrientes disponíveis aumentou muito além do esperado em solos degradados e em áreas naturais de Cerrado, conforme valores comparativos da Tabela 1 e valores expressos em Correa \& Melo Filho (1998). De acordo com a USEPA (1981), onde o tratamento de esgotos é o maior objetivo em um sistema de infiltração no solo, uma cobertura vegetal perene é desejável. Ela prolonga a vida e aumenta a eficiência do sistema, por causa da retirada de nutrientes do solo e do aumento de água evapotranspirada; portanto, a comunidade vegetal estabelecida na área certamente está cumprindo um importante papel na reciclagem de nutrientes e aumentando a eficiência e o tempo de vida desse sistema.

Em relação ao substrato anteriormente exposto, os resultados mostram que a matéria orgânica se concentrou mais nos primeiros $10 \mathrm{~cm}$ do perfil amostrado (Tabela 1, Figura 1). $\mathrm{Na}$ área irrigada, $8,1 \%$ da matéria orgânica encontram-se nos primeiros $5 \mathrm{~cm}$ da superfície, contra $1,6 \%$ sob condições naturais, porém não há diferença estatística entre os níveis de matéria orgânica das camadas $0-5 \mathrm{~cm}$ e $5-10 \mathrm{~cm}$, na área irrigada (Tabela 1). A diferença aparece entre as camadas $5-10 \mathrm{~cm} \mathrm{e}$ $10-20 \mathrm{~cm}$. A concentração de matéria orgânica nos primeiros $10 \mathrm{~cm}$ de solo é cerca de 5 vezes mais alta, quando comparada com a área controle e entre $10 \mathrm{~cm}$ e $20 \mathrm{~cm}$ de profundidade, a diferença se reduz para 2,8 vezes. A deposição excessiva de matéria orgânica na superfície de solos pode provocar colmatação e reduzir consideravelmente a taxa de infiltração. Porém isto é dependente da diferença entre as taxas de deposição e de mineralização e, caso aconteça, uma reversão

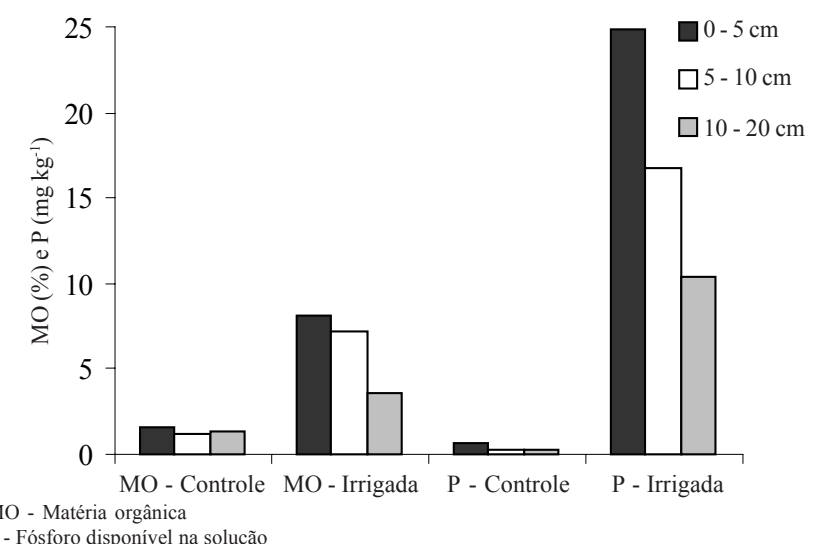

P - Fósforo disponível na solução

Figura 1. Matéria orgânica e fósforo solúvel nas áreas irrigada e de controle

Tabela 1. Matéria orgânica e concentração de nutrientes nas áreas irrigada e de controle ${ }^{1}$

\begin{tabular}{|c|c|c|c|c|c|c|c|c|}
\hline Camadas & $\mathrm{MO}^{*}$ & $\mathrm{P}$ & $\mathrm{Ca}$ & $\mathrm{Mg}$ & $\mathrm{K}$ & $\mathrm{Zn}$ & $\mathrm{Cu}$ & $\mathrm{Fe}$ \\
\hline \multirow{2}{*}{\multicolumn{9}{|c|}{ Irrigada }} \\
\hline $0-5$ & & & & $16,4 \mathrm{a}$ & $201,1 \mathrm{a}$ & $0,8 \mathrm{a}$ & $0,6 \mathrm{a}$ & $6,3 \mathrm{a}$ \\
\hline $5-10$ & $7,2 \mathrm{a}$ & $16,8 \mathrm{~b}$ & $166,9 \mathrm{~b}$ & $9,8 \mathrm{~b}$ & $174,3 \mathrm{~b}$ & $0,4 b$ & $0,7 \mathrm{~b}$ & $5,8 \mathrm{a}$ \\
\hline $10-20$ & $3,6 \mathrm{~b}$ & $10,4 \mathrm{c}$ & $39,4 \mathrm{c}$ & $3,8 \mathrm{c}$ & $156,5 \mathrm{c}$ & $0,4 \mathrm{~b}$ & $0,4 \mathrm{c}$ & $6,2 a$ \\
\hline \multicolumn{9}{|l|}{ Controle } \\
\hline $0-5$ & $1,6 \mathrm{c}$ & $0,6 \mathrm{~d}$ & $34,0 \mathrm{c}$ & $8,0 \mathrm{~d}$ & $218,3 d$ & $3,6 \mathrm{c}$ & $0,1 \mathrm{~d}$ & $45,5 \mathrm{~b}$ \\
\hline 5-10 & $1,2 \mathrm{c}$ & $0,2 \mathrm{~d}$ & $20,3 \mathrm{c}$ & $3,2 \mathrm{c}$ & $177,0 \mathrm{~b}$ & $16,6 \mathrm{~d}$ & $0,1 \mathrm{~d}$ & $41,7 \mathrm{~b}$ \\
\hline $10-20$ & $1,3 \mathrm{c}$ & $0,3 \mathrm{~d}$ & $20,4 \mathrm{c}$ & $5,1 \mathrm{e}$ & $187,5 \mathrm{a}$ & $15,6 \mathrm{~d}$ & $0,1 \mathrm{~d}$ & $23,4 \mathrm{c}$ \\
\hline
\end{tabular}

${ }^{1}$ Valores de mesma letra são estatisticamente iguais pelo teste de Tukey, a 5\% de significância

*MO - matéria orgânica 
de camadas do solo seria suficiente para diluí-la ao longo do perfil e aumentar sua taxa de mineralização.

Os valores encontrados para matéria orgânica nos primeiros $20 \mathrm{~cm}$ de solo, subtraída a quantidade de matéria orgânica presente na amostra-controle, equivalem a $10,7(+/-0,6)$ toneladas de material retido no solo e que deixaram de ser despejados no Lago Paranoá. O valor total retido em 2 anos é provavelmente mais alto, pois certamente houve prévia mineralização de parte do material depositado, anterior à coleta das amostras.

Aumento das concentrações de potássio, zinco e ferro não foi constatada na área irrigada, em comparação com a áreacontrole. As concentrações de zinco e ferro até diminuíram na área irrigada (Tabela 1) devido, provavelmente, à complexação pela matéria orgânica. Como esgotos não são fontes significativas desses elementos nem de potássio, a taxa de reposição pode estar sendo menor que a de complexação e de retirada pela vegetação; no caso do potássio, trata-se de um macronutriente altamente demandado no metabolismo e nas estruturas vegetais e, como há um desbalanço entre a sua concentracão e a de nitrogênio e fósforo em esgotos domésticos, geralmente existe a necessidade de suprimento extra de potássio em cultivos comerciais irrigados com esgoto.

A concentração de cálcio aumentou 8,6 vezes na camada $0-5 \mathrm{~cm}$ e ao longo dos $20 \mathrm{~cm}$, ela aumentou 6,8 vezes. Carraro et al. (1996) encontraram resultados semelhantes para cálcio e fósforo, quando aplicaram esgotos em um solo de textura siltosa em São Paulo. $\mathrm{O}$ aporte desses nutrientes beneficiou o crescimento da espécie plantada por eles. $\mathrm{O}$ aumento de cobre no perfil aparece em terceiro lugar, concentrando-se 6,2 vezes na camada $5-10 \mathrm{~cm}$.

$\mathrm{O}$ aumento da concentração de fósforo e cobre na área irrigada, entre $0-20 \mathrm{~cm}$ de profundidade foi, em média, de 3,9 e 5,7 vezes, respectivamente. Os níveis desses elementos na área irrigada são estatisticamente diferentes nas três profundidades definidas, cujo padrão diverge das condições naturais, onde as três camadas apresentam níveis desses elementos semelhantes (Tabela 1). Apesar de o fósforo ser relativamente imóvel no solo, ele está percolando através da matriz até, no mínimo, a profundidade de $20 \mathrm{~cm}$ amostrada (Figura 1). As concentrações de fósforo apresentam-se decrescentes da superfície para as camadas mais profundas do perfil, o que reforça esta evidência.

O fósforo aumentou 84 vezes na solução do solo na camada 5-10 cm, apesar de estar mais concentrado na camada $0-5 \mathrm{~cm}$ (Tabela 1; Figura 1); tal magnitude de concentração é a mais alta encontrada entre todos os parâmetros considerados neste trabalho. $\mathrm{O}$ aporte de fósforo proveniente do esgoto na área irrigada foi de 40,9 $\mathrm{kg}(+/-1,7 \mathrm{~kg})$, após dois anos de operação do sistema. Com a metodologia utilizada para medir o fósforo solúvel, pôde-se recuperar $3,8 \mathrm{~kg}(+/-0,2 \mathrm{~kg})$ nos primeiros 20 $\mathrm{cm}$ de substrato. Considerando-se as curvas de retenção de fósforo do solo usado neste trabalho, outros $32,3 \mathrm{~kg}(+/-1,6 \mathrm{~kg})$ encontram-se retidos no solo. Dessa forma, o total de fósforo retido no solo na data de coleta das amostras era de $36,1 \mathrm{~kg}$ $(+/-1,7 \mathrm{~kg})$. Esse valor representa $88,3 \%$ do fósforo total descarregado na área, em dois anos de operação. Essa é a eficiência do sistema implantado para a remoção de fósforo. A mensuração do fósforo retirado pelas plantas e não avaliado neste trabalho, aumentaria ainda mais essa eficiência. Culturas agrícolas são capazes de absorver entre 20 e $60 \mathrm{~kg} \mathrm{P} \mathrm{ha}^{-1}$ ano $^{-1}$ (Nucci et al.,1978).

De acordo com a USEPA (1981) os sistemas de remoção de fósforo por disposição de esgotos em solos apresentam eficiência entre 70 e $99 \%$, a depender das características químicas e físicas do substrato, do tempo de residência do esgoto no solo e de outras condições climáticas e operacionais. A eficiência de $88,3 \%$ calculada para este sistema está entre a faixa aceitável (70-99\%). Além da prática saudável em se depositar resíduos onde eles são necessários, o manejo proposto aqui também vai de encontro às necessidades preementes de se reduzir a carga orgânica e de nutrientes vertendo para cursos e reservatórios d'água.

\section{CONCLUSÕES}

As evidências e os resultados mostraram melhorias ambientais e ecológicas na área irrigada sob condições controladas. $\mathrm{O}$ estabelecimento de uma cobertura vegetal sobre solos e substratos desnudos é considerado uma das mais importantes e urgentes medidas no controle de erosão, recuperação de áreas degradadas e conservação de solos. O Sistema de Infiltração Rápida implantado na área de empréstimo abandonada mostrou-se promissor em resolver dois problemas de uma só vez: oferecer uma alternativa viável à disposição de esgotos em cursos e corpos d'água e prover as condições necessárias ao estabelecimento de uma cobertura vegetal no local. O sistema, operando sobre o horizonte $\mathrm{C}$ de um Latossolo vermelho-escuro durante 2 anos consecutivos, alcançou uma eficiência de $88,3 \%$ para a remoção de fósforo, que pode ser considerada altamente satisfatória.

\section{AGRADECIMENTOS}

Os autores agradecem à Companhia de Água e Esgoto de Brasília - CAESB, pelo auxílio na construção do Sistema de Infiltração Rápida no local de estudo.

\section{REFERÊNCIAS BIBLIOGRÁFICAS}

ASCE. Land application of wastewater - sludge. New York/USA. In: T.M. Younos (ed.) A report of the Task Committee on Land Application of Sludge of the Committee on Water Pollution Management of the Environmental Engineering Division, 1987. 89p.

CARRARO, V.J.; FIGUEIREDO, R.F.; VIEIRA, D.B. Efeito sobre as características químicas do solo pela aplicação de águas residuárias de indústria cítrica. In: CONGRESSO LATINO AMERICANO DE CIÊNCIA DO SOLO/Solo-Suelo 96, 13. 1996, Águas de Lindóia, SP: SLACS/SBCS/ESALQ/SBM. CD ROM.

CORREAA, R.S.; LEITE, L.L. Desmatamento e mineração em unidade de conservação. In: CORRÊA, R.S; MELO FILHO, B. (orgs.) Ecologia e recuperação de áreas degradadas no cerrado. Brasília. Ed. Paralelo 15, 1998. cap. 3, p.29-47. 
CORREAA, R.S.; MELO FILHO, B. Ecologia e regeneração em áreas escavadas. In: CORRÊA, R.S; MELO FILHO, B. (orgs.) Ecologia e recuperação de áreas degradadas no cerrado. Brasília. Ed. Paralelo 15, 1998. cap. 5, p. 65-99.

COURACCI FILHO, B.; SOBRINHO, P.A.; FIGUEIREDO, R.F.; VIEIRA, D.B. Avaliação da produção da matéria vegetal em solo que recebeu esgoto doméstico bruto. In: CONGRESSO LATINO AMERICANO DE CIÊNCIA DO SOLO/Solo-Suelo 96, 13. 1996, Águas de Lindóia, SP. SLACS/SBCS/ESALQ/ SBM. CD ROM.

DEDECK, R.A. Manejo e preparo do solo. In: CURSO DE RECUPERAÇÃO DE ÁREAS DEGRADADAS, V. I. Curitiba, PR: Universidade Federal do Paraná e FuPeF. p.5. 1993.

EMBRAPA. Manual de métodos de análise de solo. Rio de Janeiro: Serviço Nacional de Levantamento de Solos. Ministério da Agricultura, 1979. sp.

EPSTEIN, E. The science of composting. Lancaster, Pennsylvania, USA: Technomic Publishing Company, Inc. 1997.487p.

LOEHR, R.C. Land application of wastes. New York, USA: Vol. II van Nostrand Reinhold Environmental Engineering Series. van Nostrand Reinhold Company. 1979. 431p.

LOPES, A.S. Solos sob "cerrado": características, propriedades e manejo. Piracicaba, SP: Instituto da Potassa \& Fosfato (EUA) Instituto Internacional da Postassa (Suíça), 1983. $162 p$.

MALAVOLTA, E.; KLIEMANN, H.J. Desordens nutricionais no Cerrado. Piracicaba, SP: Instituto da Potassa \& Fosfato (EUA), 1985. 136p.

METCALF \& EDDY. Wastewater engineering. Treatment, disposal, and reuse. 3 Ed. Singapore: McGraw-Hill, Inc. International Edition, 1991. 1334p.
NUCCI, N.L.R.; COSTA e SILVA, R.J.; ARAÚJO, J.L.B. Tratamento de esgotos municipais por disposição no solo e sua aplicabilidade no Estado de São Paulo. São Paulo, SP: Fundação Prefeito Faria Lima - Centro de Estudos e Pesquisas de Administração Municipal, 1978. 70p.

REED, S.C.; CRITES, R.W.; MIDDLEBROOKS, E.J. Natural systems for waste management and treatment. 2 Ed. New York, USA: McGraw-Hill Inc., 1995.433p.

RESK, D.C. Parâmetros conservacionistas dos solos sob vegetação de cerrados. Planaltina, DF: Centro de Pesquisa Agropecuária dos Cerrados - CPAC/EMBRAPA, 1981. Circular Técnica ${ }^{\circ} 6$

SEDLAK, R.I. (ed). Phosphorus and nitrogen removal from municipal wastewater: Principles and practice. 2 Ed. New York, USA: The Soap and Detergent Association Lewis Publishers, 1991.240p.

SKILTON, J.A.; MENEZIES, N.W.; GUPPY, C.N. Phosphorus storage on effluent irrigated land. In: NATIONAL SOILS CONFERENCE, ENVIRONMENTAL BENEFITS OF SOIL MANAGEMENT. 1998, Brisbane, Austrália. Conference Proceedings, p.100-104, 1998.

USEPA - United States Environmental Protection Agency. Land treatment of municipal wastewater. EPA 625/1-81-013. Process design manual for land treatment of municipal wastewater. Cincinnati, Ohio, USA: Technology Transfer. Center for Environmental Research Information. October 1981.p.24.

USEPA - United States Environmental Protection Agency. Land treatment of municipal wastewater. Supplement on rapid infiltration and overland flow, EPA 625/1-81-013a. Cincinnati, Ohio, USA: Process design manual technology transfer. Center for Environmental Research Information. October 1984.121p. 